\title{
MONITOREO DE LA FAUNA SILVESTRE EN BOSQUE TROPICAL SECO SECUNDARIO, NANDAROLA, NANDAIME, NICARAGUA
}

\section{MONITORING OF WILDLIFE IN A SECONDARY DRY TROPICAL FOREST, NANDAROLA, NANDAIME, NICARAGUA}

\section{Garmendia-Zapata Miguel}

Docente de la Facultad de Recursos Naturales y del Ambiente, FARENA/UNA. E-mail: garmendiaz@una.edu.ni

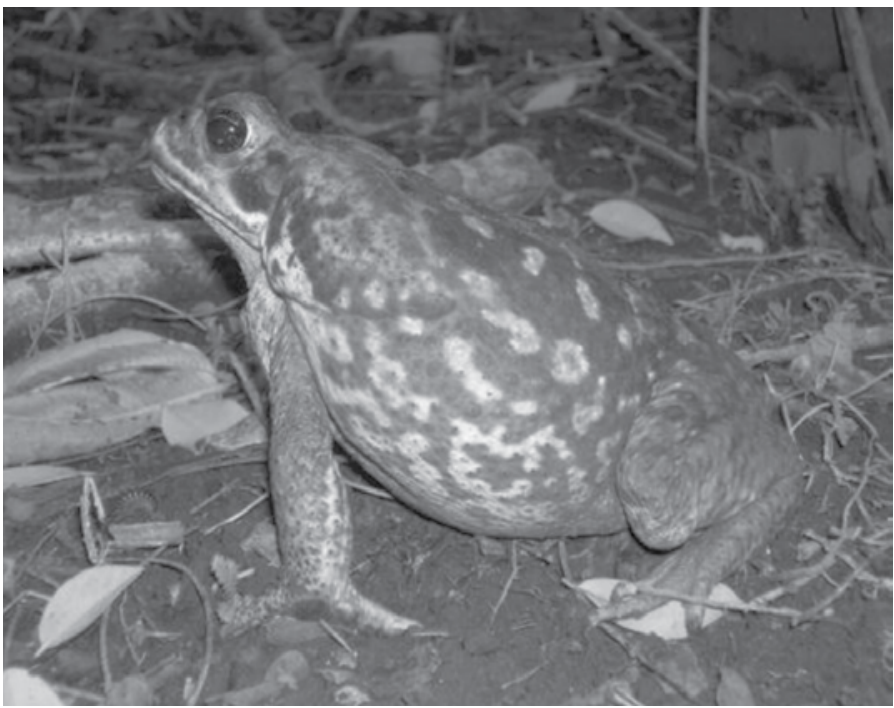

\section{RESUMEN}

Los animales silvestres obtienen refugio, hábitat, alimento y agua de la cobertura que les ofrece la vegetación dentro del bosque. En los años 60s, la mayoría de las áreas boscosas de Nandarola fueron deforestadas y usadas en la producción agrícola de granos (frijoles, maíz, arroz) y áreas ganaderas. Los campos fueron recientemente abandonados y están siendo colonizados por un joven bosque secundario (González, 2005). La fauna silvestre también sufre procesos de colonización de las áreas abandonadas, de esta forma, es importante conocer que especies son las primeras en ser parte de esa colonización y evaluar los cambios en composición de las comunidades animales en el tiempo, principalmente porque muchos vegetales dependen de éstos para realizar procesos biológicos y ecológicos tan importantes como lo son la dispersión y la polinización. Hasta el momento se han realizados dos muestreos para el monitoreo de la fauna silvestre en un parche de bosque seco secundario de 3.5 ha en Nandarola, realizándose 17 visitas en el primero (año 2007) y 15 en el segundo (año 2009), distribuidas en ocho meses (octubre-mayo en cada muestreo). El área era agrícola y fue forestal. Para la toma de datos se establecieron seis transectos 
de 20 x $100 \mathrm{~m}$ y seis puntos de conteo de $25 \mathrm{~m}$ de radio. Fueron contados e identificados todos los animales silvestres de los grupos mamíferos, aves, reptiles y anfibios mediante la técnica de avistamiento, para los cuales se calculó, se comparó la riqueza, abundancia, diversidad biológica y se describió la composición de especie, la preferencia de hábitat, los hábitos alimenticios y también se determinaron las especies de probable ocurrencia en el área de estudio. En promedio entre los dos muestreos fueron determinaron 113 individuos, agrupados en 35 especies, 34 géneros y 26 familias de fauna silvestre. De estas especies cuatro fueron mamíferos, 21 aves, siete reptiles y tres anfibios. Las familias más representadas según el número de especies fueron: Columbidae (3 especies) e Iguanidae (3). Las especies con mayor promedio de abundancia fueron: Campylorynchus rufinucha (22.5 individuos), Thryothorus pleurostictus (11.5), Calocitta formosa (10.5), Turdus grayi (8), Pachyrampuhus aglaiae (7), Eumomota supersiliosa (6.5) y Trogon melanocephalus (6). La abundancia y la riqueza no varió significativamente entre los muestreos, la diversidad fue mayor en el primero, debido al aumento de la dominancia específica (principalmente por Campylorynchus rufinucha) en el segundo muestreo. El grupo de los mamíferos, reptiles y anfibios fue poco representado en el área de estudio, pudiéndose deber a factores antropogénicos. El paisaje esta mayormente representado por especies de aves de hábitats abiertos y que se alimentan mayoritariamente de invertebrados y semillas. Por otro lado con una relación menos específica los mamíferos y reptiles fueron principalmente de hábitats generalistas y mayormente consumidores de frutas, hierbas y vertebrados. Entre las especies de probable ocurrencia en el área de estudio se determinaron: Bufo coccifer, Boa constrictor, Ctenosaura similis, Mabuya unimarginata, Amazilia rutila, Aratinga canicularis, Ciccaba virgata, Crax rubra, Polyborus plancus, Dasypus novencintus, Dasyprocta punctata, Felis pardalis, Felis wiedii, Philander opossum, Nasua narica, Odocoileus virginianus, Procyon lotor, Puma concolor, Sylvilagus sp, Tayassu tajacu, entre otros. biological diversity and describe the species composition, the habitats preference, the food habits and also the species of probable occurrence in the study area. On average across the two samples, 113 individual were identified, grouped into 35 species, 34 genera and 26 families of wildlife. Four species were mammals, 21 birds, seven reptiles and three amphibians. The most represented families according to the numbers of species were: Columbidae (3 species) and Iguanidae (3). The most represented species considering abundance were: Campylorynchus rufinucha (22.5 individuals), Thryothorus pleurostictus (11.5), Calocitta formosa (10.5), Turdus grayi (8), Pachyrampuhus aglaiae (7), Eumomota supersiliosa (6.5) and Trogon melanocephalus (6). The abundance and the richness did not vary significantly among the two samplings; the diversity was highest in the first, due to an increase of specific dominance (mainly by Campylorynchus rufinucha) in the second sampling. Mammals, reptiles and amphibians groups were little represented in the study area, it was because the surround people. The landscape was mostly represented by bird of open habitats that select invertebrate animals and seeds for feed. On the other hand, with a less specific relationship, the mammals and reptiles were mainly from general habitats and mostly consumers of fruits, grasses and vertebrates. Among the species of probable occurrence in the study we found: Bufo coccifer, Boa constrictor, Ctenosaura similis, Mabuya unimarginata, Amazilia rutila, Aratinga canicularis, Ciccaba virgata, Crax rubra, Polyborus plancus, Dasypus novencintus, Dasyprocta punctata, Felis pardalis, Felis wiedii, Philander opossum, Nasua narica, Odocoileus virginianus, Procyon lotor, Puma concolor, Sylvilagus sp, Tayassu tajacu.
$\mathrm{E}$ $60 \%$ de la población de Nicaragua esta asentada alrededor del bosque tropical seco (Sabogal \& Valerio, 1998; Anon., 2004). El bosque les provee de muchos beneficios y materiales para la construcción, madera, carpintería en general, productos no maderables, medicina, etc., pero la combinación entre el uso y la aglomeración poblacional desde hace muchos años, se ha tornado poco saludable para el mismo. El cambio de uso de la tierra de áreas boscosas a áreas agrícolas o ganadera ha sido la causa primordial de la fragmentación y pérdida de hábitats. Por naturaleza la diversidad de fauna silvestre esta íntimamente relacionada con el bosque, la mayoría de los animales silvestres obtienen refugio, hábitat, alimento y agua de la cobertura que les ofrece la vegetación, muchas especies tienen una alta especificidad en relación a la condición que requieren para su proliferación, por ejemplo, la mayoría de los mamíferos terrestres de gran tamaño requieren grandes extensiones áreas boscosas continuas, en especial se pueden mencionar a los primates, felinos, pecaris, cervidos, tapires, entre otros.

En los años 60s, la mayoría de las áreas boscosas de Nandarola fueron deforestadas y usadas en la producción agrícola de granos (frijoles, maíz, arroz) y áreas ganaderas. Los campos fueron recientemente abandonados y están siendo colonizados por un joven bosque secundario (González, 2005). La fauna silvestre también sufre un proceso de colonización de las áreas abandonadas, de esta forma, es importante conocer 
que especies son las primeras en ser parte de esa colonización y evaluar los cambios en composición de las comunidades animales en el tiempo, principalmente porque muchos vegetales dependen de éstas para realizar procesos biológicos y ecológicos tan importantes, como son la dispersión y la polinización.

\section{MATERIALES Y MÉTODOS}

Descripción del sitio de estudio. El bosque seco de Nandarola esta ubicado a siete kilómetros al sur de la ciudad de Nandaime, Granada, entre las coordenadas 11042 ' $30^{\prime \prime} \mathrm{N}$ y $86^{\circ} 05^{\prime} 30^{\prime}$ 'W. Se caracteriza por ser una zona cálida y seca con temperaturas entre $26^{\circ} \mathrm{C}$ y $29^{\circ} \mathrm{C}$ y precipitaciones que van entre 90 y $167 \mathrm{~mm}$. Las elevaciones van desde los 211 a los $261 \mathrm{msnm}$ (MARENA, 1994).

El bosque se clasifica según MARENA (1994) dentro de las formaciones forestales zonales de la región ecológica correspondiente al bosque bajo o mediano caducifolio. El área de estudio está formada por el tipo de bosque denominado tacotales. Los tacotales se forman como consecuencia del abandono de áreas agrícolas, apareciendo en las primeras etapas de la sucesión de las especies llamadas pioneras o invasoras, además se da un sotobosque de especies arbustiva.

El presente estudio se realizó en un parche de bosque seco secundario de 3.5 ha propiedad del Sr. Calos Sosa, el que hace 17 años decidió abandonar esas áreas antes dedicada a la agricultura, para que mediante la colonización natural se estableciera una nueva área forestal.

Proceso metodológico. El proceso metodológico consto del establecimiento de unidades de muestreo mediante un diseño de muestreo sistemático. Las unidades de muestreo fueron seis transectos de $20 \times 100 \mathrm{~m}$ y seis puntos de conteo de $25 \mathrm{~m}$ de radio (Figura 1). La toma de datos estaba basada principalmente en la detección de los animales mediante la técnica de avistamiento con el uso de binoculares 10x50 mm, contándose a los animales silvestres de las clases mamíferos, reptiles y anfibios (mediante los transectos) y la aves (mediante los puntos de conteo). Seguido, se realizó una revisión de literatura para fortalecer y enriquecer el estudio. Se llevaron a cavo dos conteos de la fauna silvestre, uno en el año 2007 y otro en el año 2009, para ellos se utilizaron las mismas unidades de muestreo en las mismas ubicaciones. La toma de datos se llevó a cavo por la mañana entre las 05:30-07:30 am y por la tarde entre las 04:30-06:30 pm, realizándose 17 visitas en el primer muestreo y 15 en el segundo, distribuidas en ocho meses (octubre-mayo en cada muestreo).

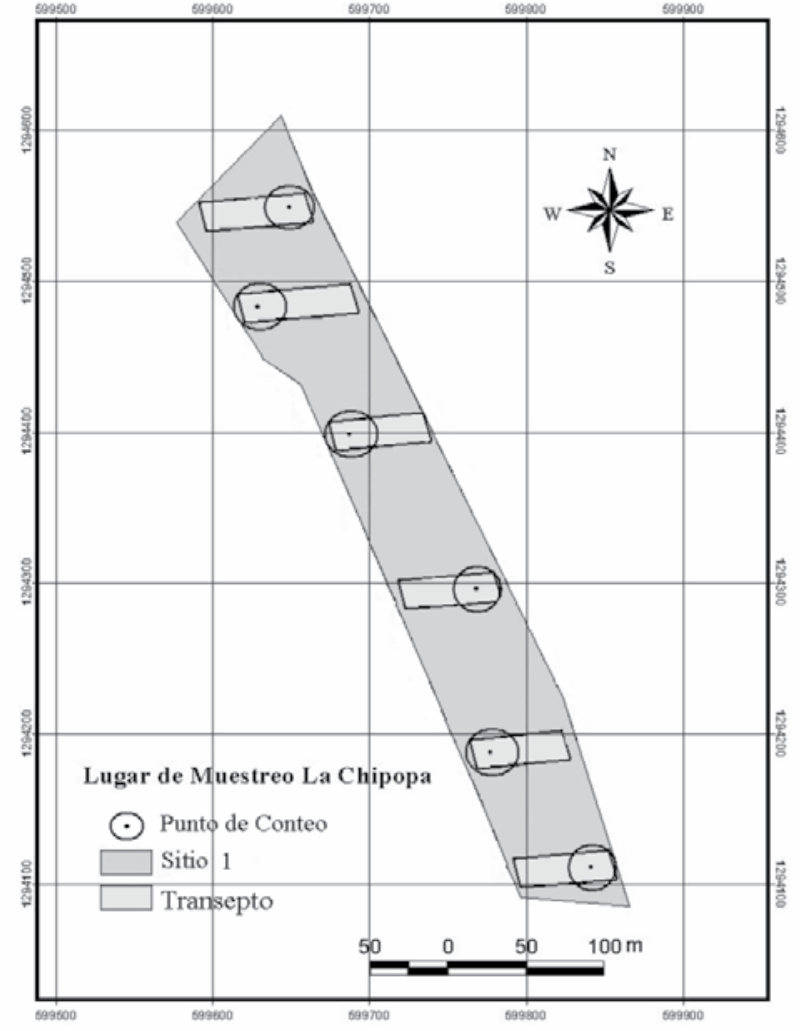

Figura 1. Representación del diseño de muestreo para el conteo de vertebrados terrestres en el área de estudio, comunidad La Chipopa.

Los animales silvestres que se encontraban dentro de las unidades muéstrales fueron identificados a especie con el apoyo de guías de campo ilustradas para vertebrados terrestres como: Neotropical Reinforest Mammals (Emmons, 1999); Mamíferos Silvestres de la Cuenca de México (Ceballos y Galindo, 1984); Bird of Costa Rica (Stiles \& Skutch, 1989); Guía Ilustrada de Herpetofauna de Nicaragua (Ruiz y Buitrago, 2003); Reptiles de Centro América (Kohler, 2003). También se contó el número de individuos para cada especie.

El análisis estaba basado en el cálculo de la abundancia, riquezay diversidad de especie (la diversidad mediante el uso de índice de Shannon-Wiener). A los datos se les aplicó prueba de normalidad de Shapiro Wilks y pruebas de inferencia principalmente: ANOVA o su homólogo no paramétrico Kruskal Wallis según el caso y Ji cuadrado como estadístico robusto. Se utilizó análisis de correspondencia como análisis multivariado y para el contraste de hipótesis de los índices de Shannon-Wiener se utilizó la prueba $t$ de Hutchenson. Todo con límite de confianza de 0.05 . Dichos cálculos se realizaron en programas de cómputo: EstimateS, Statistical Estimation of Species Richness and Shared Species from Samples Versión 7.00 (Colwell, 2004) y PAST, PAlaeontological STatistics Versión 1.29 (Hammer \& Harper, 2004). Dentro de los programas estadísticos utilizados se encuentran GraphPad InStat ${ }^{\circledR}$ 


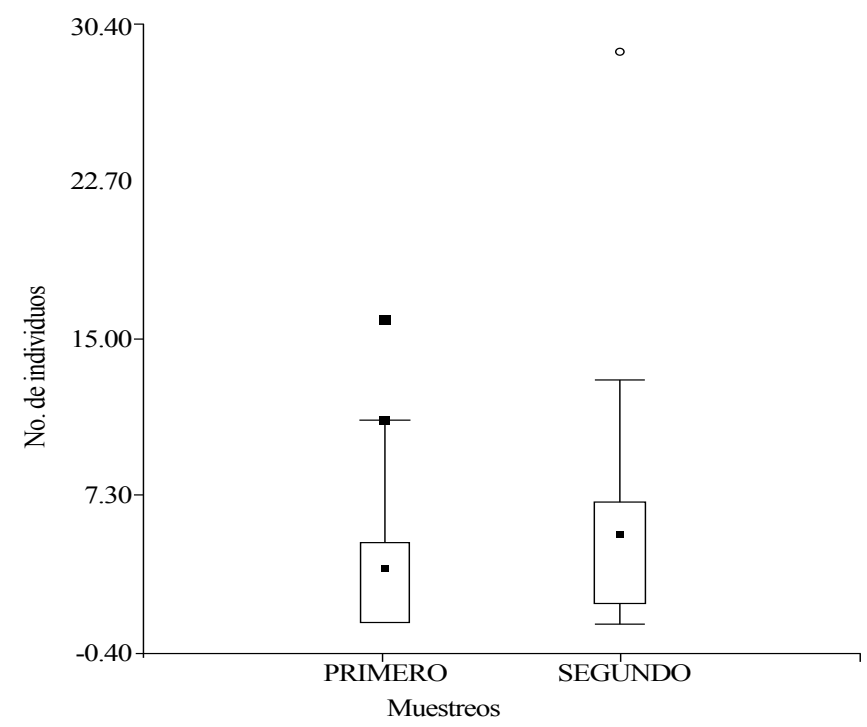

Figura 2. Gráfico boxplot de la abundancia de fauna silvestre en los dos muestreos.

Versión 3.05 (GraphPad Software, 2000) y las opciones estadísticas de PAST y Microsoft Office Excel®. Mediante revisiones bibliográficas de estudios de fauna silvestre en bosque seco, se determinaron las especies de posible ocurrencia en el área de estudio, dado a sus patrones de distribución y principalmente por ser comunes en hábitats semejantes, en un esfuerzo por determinar las especies que existían en el sitio y que futuramente podrían repoblar el área de estudio, también se le preguntó al dueño de área sobre el historial de la fauna silvestre del sitio según su experiencia.

\section{RESULTADOS Y DISCUSIÓN}

Caracterización general de la fauna silvestre en el sitio de estudio. En promedio entre los dos muestreos fueron determinaron 113 individuos, agrupados en 35 especies, 34 géneros y 26 familias de fauna silvestre. De estas especies cuatro fueron mamíferos, 21 aves, siete reptiles y tres anfibios. Las familias más representadas según el número de especies fueron: Columbidae (3 especies) e Iguanidae (3). Las especies con mayor promedio de abundancia fueron: Campylorynchus rufinucha (22.5 individuos), Thryothorus pleurostictus (11.5), Calocitta formosa (10.5), Turdus grayi (8), Pachyrampuhus aglaiae (7), Eumomota supersiliosa (6.5) y Trogon melanocephalus (6), el resto de especies están representados por cinco o menos individuos.

Comparación de la abundancia, riqueza y diversidad de especies de fauna silvestre entre los dos muestreos. Comparando las abundancias entre los dos muestreos, el segundo resultó con mayor abundancia, pero las diferencias no fueron significativas $(\mathrm{U}=552.5$, $\mathrm{p}>0.48$ ) (Figura 1). Por tanto se ha determinado que la abundancia de fauna silvestre no ha aumentado, ni disminuido considerablemente de un momento al otro.

Los datos extremos en ambos muestreos se deben a C. rufinucha, para el cual se reporta una cantidad de individuos significativamente mayor en cada muestreo $(\mathrm{X} 2=6, \mathrm{p}<0.014$ en el primero y $\mathrm{X} 2=5.67, \mathrm{p}<0.017$ en el segundo), a la vez este especie a aumentado en número de un muestreo al otro (Figura 3). C. rufinucha una especie de hábitats generalista ha sido

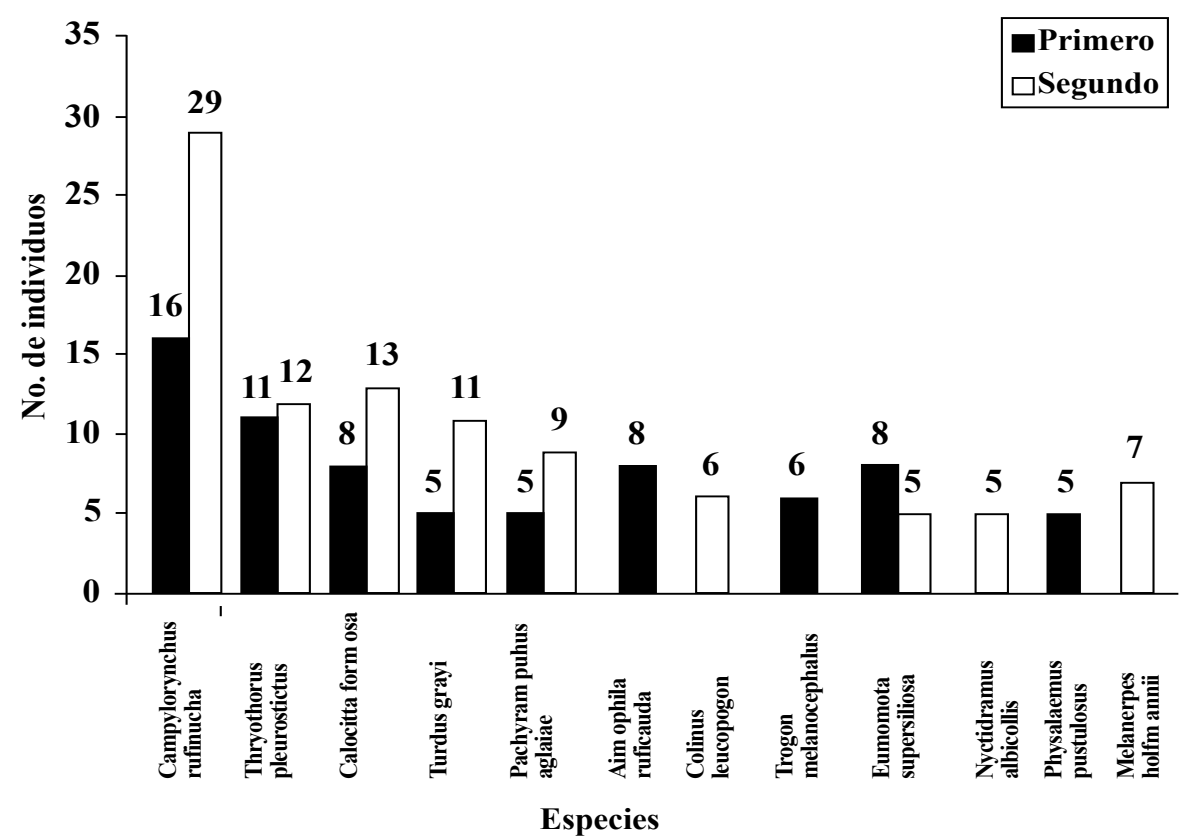

Figura 3. Especies de fauna silvestre más abundantes en los dos muestreos. 
favorecida por las condiciones que le provee el tacotal en crecimiento, principalmente por la combinación de alimentos frutos, semillas, invertebrados y por la abundancia de Corninzuelo (Acacia sp), especie favorita para la anidación de los individuos de esta especie. Las especies más abundantes después de $C$. rufinucha fueron: Thryothorus pleurostictus y Calocitta formosa (Figura $3)$, dos especies también de hábitats abiertos y que son comunes en áreas degradadas, bosque secundarias e incluso en zonas suburbanas. Es notoria la dominancia del grupo de las aves sobre el resto de los grupos.

La riqueza total de fauna silvestre fue mayor en el primer momento, pero esas diferencias resultaron no ser significativas $(\mathrm{t}=0.81, \mathrm{p}>0.44)$, por lo tanto la riqueza se mantuvo sin cambios de un momento al otro (Figura 4).

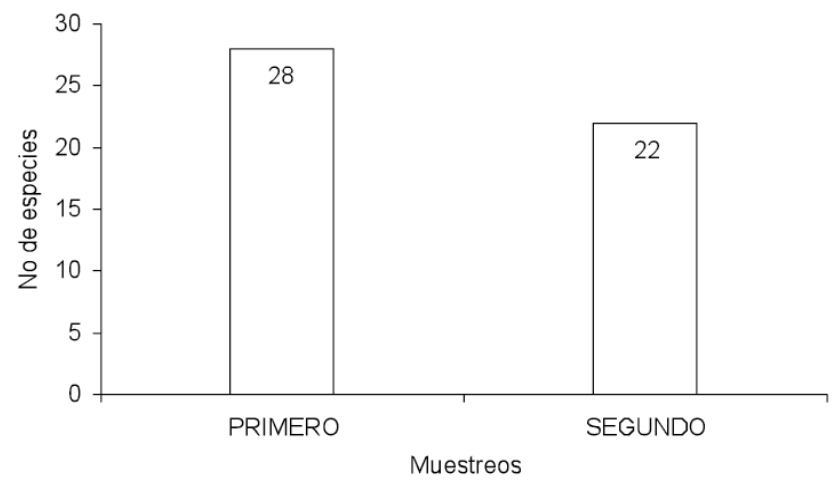

Figura 4. Riqueza total de fauna silvestre determinadas en los dos muestreos.

En la función de acumulación de especies, se observa que a medida que más individuos de fauna silvestre iban siendo registrados, el número de especies observadas incrementaba formando una curva (Figura 5), pero que no alcanzó su nivel estable o asintótico, lo que refleja que aún habían especies por determinarse con un mayor esfuerzo de muestreo, esto sucedió tanto en el primer muestreo, como en el segundo muestreo. Mediante cálculo del estimador no paramétrico Chao 2 se determinaron el número de especie que haría falta por identificar y anexar a la curva de acumulación de especie para que ésta se estabilice. El estimador refleja que para el primer muestreo, hacían falta por determinar al menos unas 23 especies más y para el segundo muestreo la curva de acumulación alcanza aún más su nivel asintótico y el estimador predice que solamente hacen falta seis especies para determinar todas las especies de fauna silvestre existentes en el sitio de estudio (Figura 5).

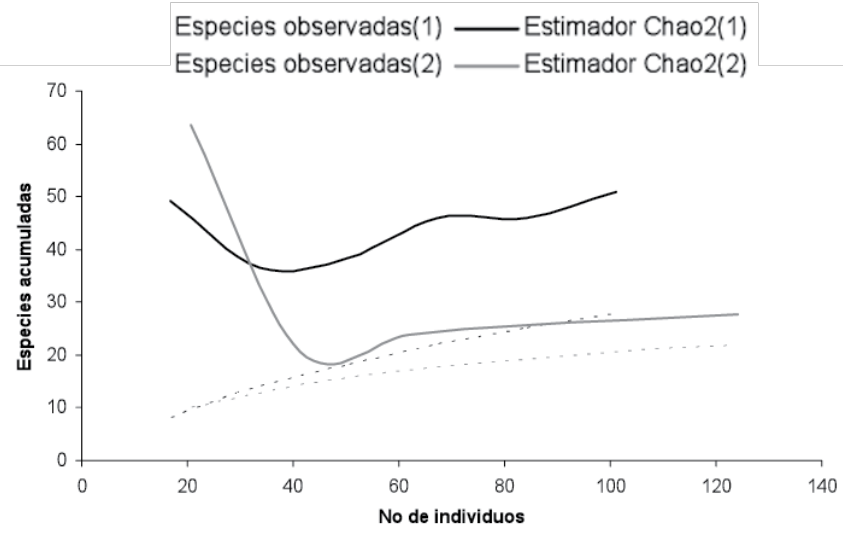

Figura 5. Curvas de acumulación para las especies observadas y estimadas según Chao2 distribuidas entre los números de individuos de fauna silvestre.

La diversidad de especie según el índice ecológico de Shannon-Wiener determina que en el primer muestreo la diversidad de fauna silvestre fue significativamente mayor $(\mathrm{t}=2.52, \mathrm{p}<0.012)$ comparado con el segundo muestreo (Tabla 1).

Tabla 1. Especies observadas, esperadas e índice de diversidad de ShannonWiener para los dos momentos de muestreo

\begin{tabular}{lll}
\hline & Primero & Segundo \\
\hline Especies observadas & 28 & 22 \\
Especies esperadas & 51 & 28 \\
Diversidad & 2.98 & 2.61 \\
\hline
\end{tabular}

Dichas disparidad pueden haber sido provocada por el aumento de las especies más abundantes (C. rufinucha, $T$. pleurostictus y C.formosa), en especial de C. rufinucha (Figura 2 y Figura 3) que reducen la equitatividad de la distribución del número de individuos por especie en el segundo muestreo y por lo tanto reduce el valor del índice en éste mismo.

\section{Comparación de la composición de especie de fauna silvestre por clase}

Composición de mamíferos. Solamente se determinaron cuatro especies de mamíferos, tres en el primero y dos en el segundo muestreo, de estas especies Coendou prehensilis fue el que se determinó en los dos. Potos flavus y Sciurus variegatoides solamente de determinaron en el primer momento y Sylvilagus sp solamente en el segundo.

La riqueza de mamíferos en general resultó ser muy baja comparada con los resultados de (Espinales, 2003) en la Reserva Natural Península de Cosigüina, en donde se determinaron 19 especies de mamíferos (sin incluir los roedores y quirópteros). En notoria la ausencia de muchas especies de mamíferos medianos y grandes propios del bosque seco que de una forma u otra, 
aún no se observan presente dentro del área de estudio, tales como caninos, felinos, agutides, dasyproctides, tayassides y odocoileus.

Lo anterior puede que se deba a la antes descrita "caza por placer" de los pobladores. Por otra parte el aislamiento geográfico, ya que el área abandonada está rodeada de áreas agrícolas sin colindar con otro parche de bosque. Es importante señalar que la comunidad de mamíferos es una de las más afectadas en los sitios donde la presencia humana es persistente. Todas las especies encontradas en el área de estudio son de hábitos generalistas o prefieren sitios abiertos en cuanto a hábitat y son fácilmente observables en bosques secundarios y ahora se ha determinado que se encuentran dentro de las especies colonizadoras de esta área en abandono.

Lo particular del sitio es que la existencia de senderos y pasaderos de gente posiblemente sea la causa de los desbalances en abundancia y riqueza entre los dos momentos, y también sea la causa de la reducida cantidad de mamíferos determinados. En campo fueron observados niños con tiradoras y perros. Mediante entrevistas a propietarios, se ha descubierto que existe falta de cultura ambientalista entre los vecinos aledaños al área en abandono. Muchos de estos dan la muerte a mamíferos (incluyendo a las cuatro especies determinadas en este monitoreo) sin necesidad alguna, o sea, sin brindarles un uso, sencillamente los animales son sacrificados por placer.

Composición de aves. El grupo de las aves fue el que mayor riqueza y abundancia registró, determinándose 21 especies lo que representa un $3 \%$ de las especies de Nicaragua. Las especies más representativas de este grupo, según el número de individuos fueron: $C$. rufinucha, T. pleurostictus, C. formosa, Turdus grayi, Pachyrampuhus aglaiae, Eumomota supersiliosa $y$ Trogon melanocephalus, en el caso de los cuatro primeros e incluyendo a E. supersiliosa son especies que se frecuentan hábitats abiertos y zonas urbanas, sin especificidad en hábitat (Stiles \& Skutch, 1989), son frecuentes en charrales, tacotales, sitios abandonados y bosque secundarios. En un estudios de diversidad de aves en bosque fragmentados elaborado por Vilchez, et. al (2004) en Rivas, se determinan estas especies como las de mayor abundancia en esos tipo de paisajes, y fueron registradas desde los parches más pequeños, hasta los más grandes.

Una limitante para el aumento de la riqueza de aves en el sitio de estudio, es el tamaño de la misma área, la cual es muy pequeña (3.5 ha). Gillespie (2000) señala que "la diversidad de aves en los bosques secos secundarios de Centro América estaba determinada por los tamaños de los parches de bosque".

P. aglaiae $y \quad T$. melanocephalus son más específicas de bosques secundarios y zonas alteradas, pero pocas veces se les encuentra frecuentando áreas urbanas y sitios totalmente abiertos. Estas especies más abundantes son potenciales consumidoras de insectos y no esta esclarecida una importancia ecológica significativa de éste tipo de hábito alimenticio para con el desarrollo del área abandonada, a como lo hacen otras especies que fueron determinadas en menores cantidades como: Amazona auropalliata, Columba flavirostris, Columbina inca y Leptotila verreavxi mediante la dispersión de semillas y frutos.

En el segundo muestreo la abundancia de aves aumento de 78 a 116 individuos, pero esas diferencias no fueron estadísticamente significativas $(\mathrm{t}=-1.038$, $p>0.306$ ). La riqueza se mantuvo igual en los dos sitios y la diversidad de aves fue de 2.50 en el primer período $\mathrm{y}$ de 2.43 en el segundo, dichos cambios tampoco son significativos $(\mathrm{t}=0.29, \mathrm{p}>0.77)$.

Una de las especies de aves registrada en el segundo muestreo se clasifican como indicadora de ecosistemas, ésta fue: Amazona auropalliata, dado a sus requerimientos altamente específicos (Espinales, 2003).

Composición de reptiles. En los recorridos fueron determinadas siete especies de reptiles, representando el $3.5 \%$ de los reptiles de Nicaragua. Las especies más representadas según el número de individuos fueron: Ameiva quadrilineata y Rhinochemmys pulcherrima, ambas con tres individuos. Es determinante que el número de reptiles en el área de estudio es muy bajo, pese a que se hiso un gran esfuerzo en la búsqueda de estos individuos. La primer especie es común, propia de estas zonas (Köhler, 2003), no tiene preferencias de hábitat y se puede encontrar en cualquier sitios incluso dentro y alrededor de las viviendas de los pobladores. $R$. pulcherrima es otra especie común, principalmente cerca de los cuerpos de agua y zonas temporalmente inundadas. Ambas especies solo se registraron en el primer período de muestreo. Los reptiles al igual que los mamíferos son victimas de la cultura de los pobladores, el hecho de ser animales que tienen sus hábitats y nichos ecológicos en o cerca del suelo, contrario a la mayoría de las aves, les hace ser presa fácil de las personas que matan animales por placer. En los recorridos dentro del área de estudio se observó directamente a personas matando a una serpiente de la especie Conophis lineatus, alegando que es peligrosa por su letal mordida, la especie es venenosa, pero no es mortal (Köhler, 2003). En otra ocasión dos jóvenes de la comunidad nos mostraron una víbora de la especie Crotalus durissus la cual fue muerta tras una exhaustiva persecución una vez que fue avistada por ellos mismos.

Notoria es la diferencia entre los dos períodos de muestreo, siendo el primero en que posee una mayor riqueza y abundancia comparada con el segundo (riqueza: 6 y 1 ; abundancia: 11 y 2 respectivamente). Solamente 
Norops limifrons fue determinado en el segundo conteo. Pese a que los dos muestreos se realizaron con iguales esfuerzos y en las mismas épocas, no se puede alegar de una forma directa las causas de las reducciones de un período a otro, ya que será necesario complementar la información con datos de otros muestreos, es necesario determinar cuántos oscilan dichas riquezas y abundancias en el tiempo, por lo pronto solo se puede especular que la reducción se puede deber a las intervenciones humanas, resumidas en ataques directo a los animales o al ataque de los perros.

Diversidad de anfibios. Fueron determinadas solamente tres especies de anfibios: Bufo marinus en los dos muestreos, Bufo valliceps en el segundo y Physalaemus pustulosus en el primero. La riqueza fue igual para ambos períodos pero la abundancia fue mayor en el primero ( 6 y 4 respectivamente). B. marinus es una especie que se encuentra en todo el país y no es nada raro haberla encontrado en este sitio y en los dos períodos. $P$. pustulosus si es más probable encontrarla en sabanas o bosques deciduos y cuerpos de agua al igual que B. valliceps (Ruiz y Buitrago, 2003).

Preferencia de hábitats y gremios alimenticios. Las aves estaban relacionadas de una forma muy estrecha con los hábitats abiertos, dado a que el área de estudio es pequeña y que está rodada por áreas abiertas agrícolas y parches de bosque aislados. A la vez, las aves prefieren alimentarse casi exclusivamente de invertebrados y de granos, este último hábito alimenticio es de singular importancia para la dispersión de semillas de diferentes especies vegetales. El resto de los grupos (mamíferos, reptiles y anfibios) no parece tener exclusiva preferencia por algún tipo de hábitat, ni con ninguno de los gremios alimenticios, pero si es evidente que los mamíferos $\mathrm{y}$ reptiles tienden a preferir hábitats generalistas y a consumir más frutos, hierbas y vertebrados (Figura 6).

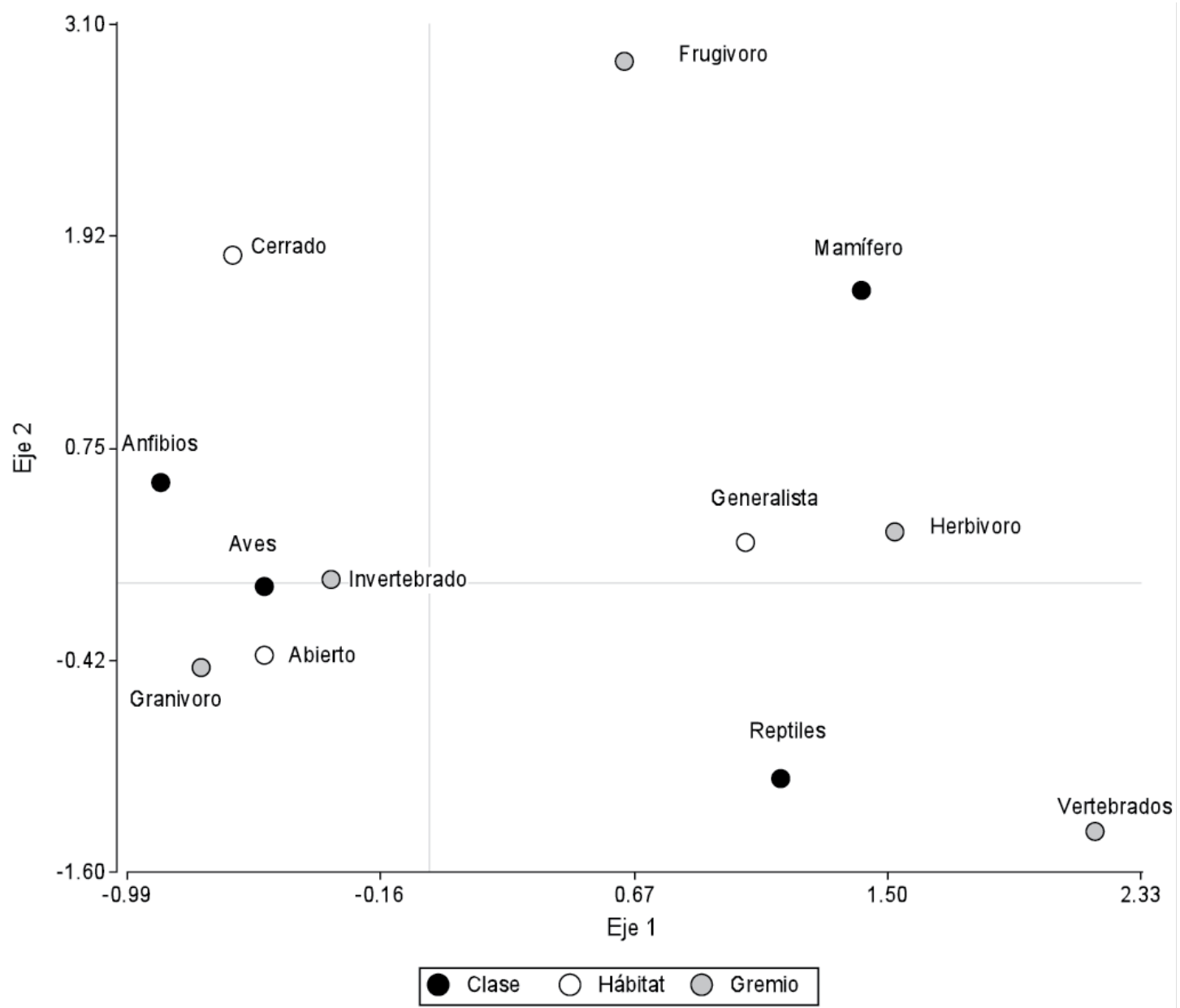

Figura 6. Bi-plot que muestra el resultado del análisis de correspondencia de la preferencia de hábitat y gremios alimenticios para cada clase. 
Es fundamental destacar que las preferencias de hábitats y gremios alimenticios son contrastantes entre los grupos de fauna silvestre, por un lado las aves y lo anfibios tienen requerimientos opuestos a los mamíferos y reptiles, en el bi-plot de la figura 6 se observa la separación en el eje de las accisas, entre los dualismos aves-anfibios con sus requerimientos de hábitats y gremios alimenticios diferentes a los mamíferos-reptiles, las relaciones son significativas $(\mathrm{X} 2=46.67, \mathrm{p}<0.027)$.

\section{Especies de probable ocurrencia en el área de estudio}

Ha como ya se expreso anteriormente, el área de estudio de Nandarola tiene una relativa poca riqueza de especie de fauna silvestre, existe posibilidad de que en el futuro, animales silvestres proliferen en el sitio, entre las especies que no se determinaron en el área, pero que tengan potencial el la colonización se encuentran:

Tabla 2. Lista de especies de probable ocurrencia en el área de estudio

\begin{tabular}{|c|c|c|c|}
\hline Clase & Especie & Clase & Especie \\
\hline Amphibia & $\begin{array}{l}\text { Bufo coccifer } \\
\text { Smilisca baudinii }\end{array}$ & Aves & $\begin{array}{l}\text { Myiodynastes luteiventris } \\
\text { Ortalis leucogastra }\end{array}$ \\
\hline Reptilia & $\begin{array}{l}\text { Basiliscus sp } \\
\text { Boa constrictor } \\
\text { Ctenosaura similis } \\
\text { Mabuya unimarginata } \\
\text { Oxibelis sp } \\
\text { Sceloporus carinatus }\end{array}$ & & $\begin{array}{l}\text { Otus cooperi } \\
\text { Pachyranphus sp } \\
\text { Polyborus plancus } \\
\text { Pteroglossus torquagus } \\
\text { Thraupis episcopus } \\
\text { Tyrannus forficatus }\end{array}$ \\
\hline Aves & $\begin{array}{l}\text { Accipiter sp } \\
\text { Amazilia rutila } \\
\text { Aratinga canicularis } \\
\text { Brotogeris jugularis } \\
\text { Buteo sp } \\
\text { Campephilus guatemalinsis } \\
\text { Ciccaba } \\
\text { Ciccaba virgata } \\
\text { Crax rubra } \\
\text { Empidonax sp } \\
\text { Geotrygon montana } \\
\text { Hirundo rustica } \\
\text { Megarynchus pitangua } \\
\text { Morococcyx erythropygus }\end{array}$ & Mamíferos & $\begin{array}{l}\text { Vermivora } \mathrm{sp} \\
\text { Canis latrans } \\
\text { Conepatus semistriatus } \\
\text { Dasyprocta punctata } \\
\text { Dasypus novencintus } \\
\text { Felis pardalis } \\
\text { Felis wiedii } \\
\text { Philander opossum } \\
\text { Nasua narica } \\
\text { Odocoileus virginianus } \\
\text { Procyon lotor } \\
\text { Puma concolor } \\
\text { Sylvilagus } \mathrm{sp} \\
\text { Tayassu tajacu }\end{array}$ \\
\hline
\end{tabular}

\section{CONCLUSIONES}

Se determinaron en promedio 113 individuos, agrupados en 35 especies, 34 géneros y 26 familias de fauna silvestre. De estas especies cuatro fueron mamíferos, 21 aves, siete reptiles y tres anfibios.

Las especies más abundantes fueron: Campylorynchus rufinucha, Thryothorus pleurostictus, Calocitta formosa y Turdus grayi.

La abundancia y la riqueza no ha variado considerablemente del primer muestreo al segundo, la diversidad cambio favoreciendo al primer momento, dado al aumento de la dominancia específica (dado al aumento de $C$. rufinucha) en el segundo muestreo.

El grupo de los mamíferos, reptiles y anfibios fue poco representado en el área de estudio, pudiéndose deber a factores antropogénicos. Siendo obvia la poca presencia de mamíferos de tamaño mediano y grandes. El paisaje esta mayormente representado por especies de aves de hábitats abiertos y que se alimentan mayoritariamente de invertebrados y semillas. Por otro lado con una relación menos específica los mamíferos y reptiles fueron principalmente de hábitats generalistas y mayormente consumidores de frutas, hierbas y vertebrados.

Entre las especies de probable ocurrencia en el área de estudio se determinaron: Bufo coccifer, Boa constrictor, Ctenosaura similis, Mabuya unimarginata, Amazilia rutila, Aratinga canicularis, Ciccaba virgata, Crax rubra, Polyborus plancus, Dasypus novencintus, Dasyprocta punctata, Felis pardalis, Felis wiedii, Philander opossum, Nasua narica, Odocoileus virginianus, Procyon lotor, Puma concolor, Sylvilagus sp, Tayassu tajacu, entre otros. 


\section{REFERENCIAS BIBIOGRÁFICAS}

ANON. 2004. Estado del Ambiente en Nicaragua 2003. II Informe GEO. Gobierno de Nicaragua. Ministerio del Ambiente y los Recursos Naturales.

Colwell, RK. 2004. EstimateS, Statistical Estimation of Species Richness and Shared Species from Samples. (programa de cómputo) Versión 7.00. University of Connecticut. United States.

Gillespie, TW. (2001). Patterns of Vertebrate Species Richness and Conservation in Nicaragua. Natural Areas Association. USA. Natural Areas Journal 21:159-167.

González, B. 2005. Tree species diversity and regeneration of tropical dry forest in Nicaragua. Swedish University of Agricultural Sciences. SLU 2005:58

Hammer, Ø; Harper, DAT. 2004. PAST Palaeontological Statistics. (programa de cómputo) Versión 1.29. University of Oslo, Noruega.

Kohler, G. 2003. Reptiles de Centro América. Offerbach; herpeton Verlog, ISBN. 367P.

MARENA (Ministerio de Recursos Naturales y del Ambiente, NI). 1994. Proyecto Nandarola/ MARENADED, Plan de manejo de la cooperativa Pedro Joaquin Chamorro Cardenal. Proyecto de protección del bosque. Managua, Nicaragua. $35 \mathrm{p}$.

Martínez-Sánchez, J; Saldaña T, O. 1985. Fauna del complejo volcánico Asososca-Momotombo, departamento de León, Nicaragua; informe preliminar. Museo de Zoología, IRENA, Managua, Nicaragua. 3 p.

Martínez-Sánchez, J; Saldaña T, O. 1985. Fauna de la península de Chiltepe, departamento de Managua, Nicaragua. Museo de Zoología, IRENA, Managua, Nicaragua. 35 p.

Martínez-Sánchez, J; Saldaña T, O. 1985. Fauna del volcán San Cristóbal. Museo de Zoología, IRENA, Managua, Nicaragua. $11 \mathrm{p}$.

Martínez - Sánchez, JC. 2001. Biodiversidad zoológica en Nicaragua. Una estrategia nacional para su conservación. MARENA/PNUD. Managua, Nicaragua. $174 \mathrm{p}$.

Ruiz, GA; Buitrago, F. 2003. Guía ilustrada de la Herpetofauna de Nicaragua. Primera edición. ARAUCARIAMARENA-AECI. Managua, Nicaragua. 337 p.

Vilchez, S. 2004. Diversidad de aves en un paisaje fragmentado de bosque seco en Rivas, Nicaragua. Fundación Nicaragüense para la Conservación (Fundación Cocibolca). Centro Agronómico Tropical de Investigación y Enseñanza (CATIE). Disponible en: http://bibliotecavirtual.clacso.org.ar/ar/libros/ nicargua/uca/encuen/encuen68/art4.rtf 